\title{
Robotic liver surgery: literature review and current evidence
}

\author{
Andrea Ruzzenente, Laura Alaimo, Simone Conci, Fabio Bagante, Tommaso Campagnaro, Corrado \\ Pedrazzani, Alfredo Guglielmi
}

Department of Surgery, Unit of Hepato-Pancreato-Biliary Surgery, Verona 37134, Italy.

Correspondence to: Dr. Andrea Ruzzenente, Department of Surgery, Unit of Hepato-Pancreato-Biliary Surgery, G.B. Rossi Hospital, University of Verona, Verona 37134, Italy. E-mail: andrea.ruzzenente@univr.it

How to cite this article: Ruzzenente A, Alaimo L, Conci S, Bagante F, Campagnaro T, Pedrazzani C, Guglielmi A. Robotic liver surgery: literature review and current evidence. Mini-invasive Surg 2020;4:91. http://dx.doi.org/10.20517/2574-1225.2020.90

Received: 20 Sep 2020 First Decision: 28 Oct 2020 Revised: 27 Nov 2020 Accepted: 1 Dec 2020 Published: 19 Dec 2020

Academic Editor: Giulio Belli Copy Editor: Cai-Hong Wang Production Editor: Jing Yu

\begin{abstract}
In the field of minimally invasive surgery, robotic surgery (RS) was introduced to overcome drawbacks in laparoscopic surgery. However, its clinical application in hepatobiliary surgery is not yet standardized. This review analyzed the results of RS to clarify the benefits of robotic liver surgery in comparison with standard laparoscopy. Among 112 publications found in the literature, the 72 most relevant were selected and the following data were extracted: patients characteristics, operative procedures, histopathology, short-term and long-term outcomes, and costs. Twenty-nine articles on robotic liver resections, published in the last five years (2015-2020) and including 1831 patients, were analyzed. Twenty-five comparative studies between robotic and laparoscopic surgery were evaluated to underline the differences in operative outcomes. Eventually, 4 sub-group analyses were conducted on hepatocellular carcinoma, gallbladder cancer, hilar cholangiocarcinoma, and colorectal liver metastases. Almost all the authors reported data on safety, feasibility and oncologic effectiveness of RS reaching comparable results with laparoscopy. However, even if robotic surgery showed longer operative time and higher costs, in selected cases it allowed to increase the rate of minimally invasive approach when compared with laparoscopy. Thus, both open and minimally invasive surgery should be provided in a modern hepatobiliary center, including the robotic approach particularly to complex cases, otherwise very demanding by laparoscopy. In conclusion, different techniques should be tailored to each patient, choosing the minimally invasive approach when possible, enhancing patients' recovery after surgery, especially in cirrhotic livers and in the context of liver transplantation. Although many centers experienced robotic liver surgery, more and larger studies are necessary to define its real benefits relative to laparoscopy, in order to standardize patient selection criteria and techniques.
\end{abstract}

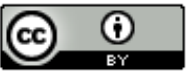

(C) The Author(s) 2020. Open Access This article is licensed under a Creative Commons Attribution 4.0 International License (https://creativecommons.org/licenses/by/4.0/), which permits unrestricted use, sharing, adaptation, distribution and reproduction in any medium or format, for any purpose, even commercially, as long as you give appropriate credit to the original author(s) and the source, provide a link to the Creative Commons license, and indicate if changes were made.

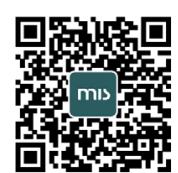


Keywords: Robotic liver surgery, robotic liver resections, laparoscopic liver resections, hepatocellular carcinoma, cholangiocarcinoma, gallbladder cancer, colorectal liver metastases

\section{INTRODUCTION}

Since its introduction, robotic surgery (RS) has received great interest from scientific societies. In the era of minimally invasive surgery (MIS) it represents an advanced technique able to overcome some limitations of laparoscopy. Nevertheless, its use is not standardized in hepatobiliary surgery. Although single surgeons and centers have published their experiences demonstrating the safety and feasibility of the technique, large international studies are limited and few publications reported long-term outcomes. The advantages of RS are several: it provides increased surgical dexterity and enhanced suturing ability, thanks to a magnified three-dimensional view of the operative field, hand tremor filtration and articulating instruments with seven-degrees of freedom. Furthermore, this approach reduces significantly surgeons' fatigue, improving performances for long operations ${ }^{[1,2]}$. In addition, RS supports and upgrades the technology of specific surgical tools, that can help surgeons to face challenging situations and improve surgical results, such as with intraoperative ultrasound, near-infrared fluorescence with indocyanine green, CT and MR images integrated into the robotic console. The images can be simultaneously displayed with the operative field during liver parenchymal transection, allowing the surgeon to change the previously marked transection line if necessary and to detect further lesions, gaining adequate margins for malignancies ${ }^{[3-5]}$. On the contrary, current RS systems' disadvantages include the absence of a dedicated instrument for transection (i.e., CUSA), the need for additional surgeons and time for instrument replacement, the learning curve of the team to dock the instruments and the lack of haptic feedback ${ }^{[2]}$. Nonetheless, the development of skills and experience of the surgical team can significantly decrease the length of RS associated to the docking time and the replacement of the instruments ${ }^{[6]}$. Finally, one of the major drawbacks of RS are costs, limiting its use to selected surgical procedures and few centers. As a minimally invasive approach, RS allows improvement of almost all the parameters of postoperative recovery, such as pain control, oral intake, postoperative morbidity and length of hospital stay ${ }^{[7]}$. Recently, the Southampton international guidelines, providing indications and limits of liver MIS, advocated RS as a promising, but not yet standardized, $\operatorname{approach}^{[8]}$. The aim of this review was to analyze the results of robotic hepatobiliary surgery and to compare them with laparoscopy, in order to clarify the benefits and contraindications of RS.

\section{METHODS}

A search of the current literature on robotic liver surgery was conducted in PubMed, Medline, PMC and Google Scholar databases. The research terms adopted were: robotic/robot-assisted liver surgery/ resection, hepatic robotic surgery/resection, robotic/robot-assisted hepatectomies. Only articles published in English were selected. Further reports were retrieved from those listed in the articles' references and from the manual search on specific additional topics, such as robotic surgery for hepatocellular carcinoma, cholangiocarcinoma, gallbladder cancer, colorectal liver metastases, lesions located in postero-superior liver segments, comparison between laparoscopic and robotic hepatic resections.

Among the 112 publications analyzed, the most significant were selected according to the following factors: quality of data reported and of statistical analysis adopted, relevance in scientific literature, date of publication. In case of overlapping studies with the same first author, the most recent was chosen. Once reviews, meta-analyses and studies reporting incomplete or unclear information were excluded, the following data were extracted from the 72 remaining publications: patient characteristics (number of patients, age, sex, body mass index, ASA score, comorbidities, previous chemotherapy and abdominal surgery), operative procedure (type of resection, use of Pringle maneuver, additional simultaneous procedures, intraoperative drain placement, estimated blood loss, operation time, 


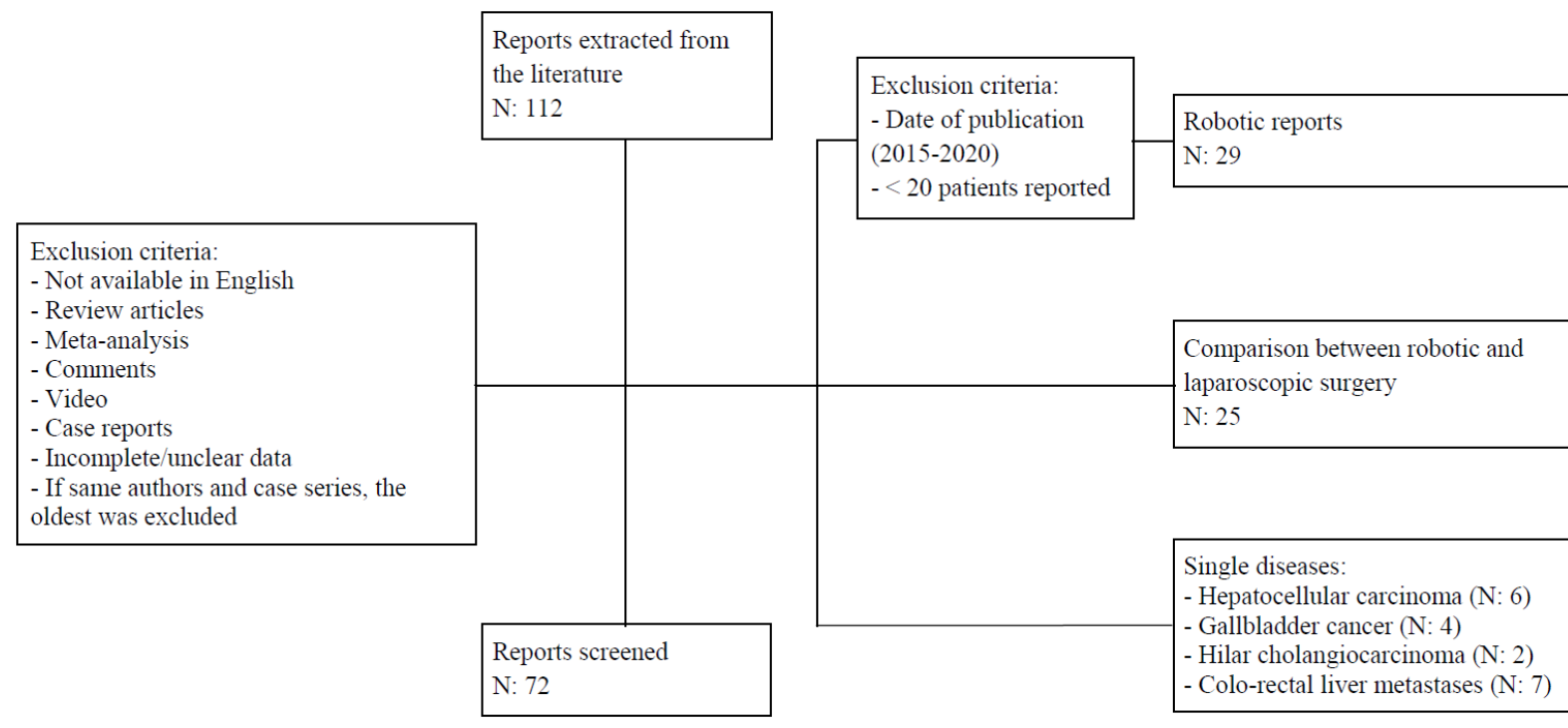

Figure 1. Selection of articles

conversion rate), histopathology (nature of the lesion, median tumor size, number of lesions, margin status, lymphadenectomy), short-term outcomes (overall morbidity, major complications, perioperative blood transfusions, admission to intensive care units, length of hospital stay, surgery-related readmission, reoperation within 30 days, 30- and 90-days mortality), long-term outcomes (disease free survival, overall survival), costs [Figure 1].

Minor and major resections were defined according to the Brisbane 2000 Terminology of Liver Anatomy and Resections ${ }^{[9]}$. The Clavien-Dindo Classification of surgical complications was adopted to define major complications as grade three or more ${ }^{[10]}$. Firstly, 29 publications on robotic liver surgery were selected and reviewed, excluding those reporting less than 20 patients. Secondly, 25 articles comparing robotic and laparoscopic liver resections were reviewed. Eventually, 4 sub-group analyses were conducted including studies on single malignant hepatobiliary diseases: hepatocellular carcinoma (HCC), gallbladder cancer (GBC), hilar cholangiocarcinoma (hCCC), colorectal liver metastases (CRLM).

\section{RESULTS}

\section{Robotic liver surgery}

Twenty-nine articles, published in the last five years (2015-2020) including a number of patients greater than or equal to 20 , were analyzed [Table 1]. The total number of patients reported in 29 studies was 1831 , with a median number of 61 patients (range 20-183). The median age was 61 years old (range 45-69.4). All the studies were retrospective and most of them reported cumulative results, without any differentiation between benign and malignant diseases or minor and major liver resections.

\section{Type of liver resection}

Referring to the type of resections, 1328 (69.5\%) were minor, and 584 (30.5\%) were major resections. The number of "technically major resections" ${ }^{\text {"[8] }}$ (segments $\left.1,4 \mathrm{a}, 7,8\right)$ collected was 214 (11.7\%). The studies including resections of these segments reported a longer operative time and greater estimated blood loss (EBL). Nota et al. ${ }^{[11]}$ published a multi-institutional propensity score study (31 matchings), demonstrating that minor robotic resections of postero-superior segments were safe and feasible, improving outcomes in comparison with open surgery [median EBL $180 \mathrm{~mL} v s .300 \mathrm{~mL}$, operative time $198 \mathrm{~min} v s .255 \mathrm{~min}$, length of stay (LOS) 4 days vs. 10 days]. 
Table 1. Robotic liver surgery

\begin{tabular}{|c|c|c|c|c|c|c|c|c|c|c|c|}
\hline Authors & Cases & Age & Location & $\begin{array}{l}\text { Major/ } \\
\text { minor }\end{array}$ & EBL & Time & Conversion & Malignant & RO & LoS & $\begin{array}{l}\text { Overall/Major } \\
\text { complications }\end{array}$ \\
\hline Chong et al. ${ }^{[1]}$ & 91 & 58.7 & LS, Sg1 & $19 / 72$ & $274.6^{\star}$ & $259.3^{*}$ & 7.7 & 100 & 98.9 & 4.8 & $9.9 / 3.3$ \\
\hline Montalti et al. ${ }^{[2]}$ & 36 & 62 & PS & $0 / 36$ & $415^{\star}$ & $306^{\star}$ & 13.9 & 69.4 & 89 & $6^{\star}$ & $19.4 / 11.1$ \\
\hline Marino et al..$^{[4]}$ & 40 & 69.4 & LS & $18 / 22$ & 260 & 305 & 2.5 & 100 & 100 & 7.4 & $20 / 12.5$ \\
\hline Pesi et al. ${ }^{[5]}$ & 51 & 63 & LS, PS & $13 / 38$ & 100 & 300 & 2 & 100 & 100 & 5 & $18 / 9.8$ \\
\hline Magistri et al. ${ }^{[6]}$ & 22 & 60.8 & LS & $2 / 20$ & 400 & $318^{*}$ & 0 & 100 & 95.5 & 5.1 & $68.2 / 9$ \\
\hline Nota et al. ${ }^{[11]}$ & 51 & 59 & PS & $0 / 51$ & 180 & 198 & 8 & 88.2 & 84 & 4 & $-/ 6$ \\
\hline Daskalaki et al. ${ }^{[12]}$ & 67 & 52.5 & LS & $29 / 39$ & $438^{\star}$ & $293.4^{*}$ & 8.8 & 55.8 & - & $6.8^{*}$ & $22 / 4.4$ \\
\hline Hu et al. ${ }^{[13]}$ & 58 & 52.2 & LS & $0 / 58$ & $80.1^{\star}$ & $107^{\star}$ & 0 & 62 & 100 & 4.3 & $1.7 /-$ \\
\hline Lee et al. ${ }^{[14]}$ & 70 & 58 & LS & $14 / 56$ & 100 & 251.5 & 5.7 & 74.2 & 98.2 & 5 & $11.4 /-$ \\
\hline Felli et al. ${ }^{[15]}$ & 20 & 64.6 & LS, PS & $2 / 18$ & 50 & $141.5^{\star}$ & 0 & 85 & - & - & - \\
\hline Lai et al. ${ }^{[16]}$ & 95 & 62.1 & LS, PS & $27 / 75$ & $334.6^{\star}$ & 207.4 & 4 & 100 & 96 & $7.3^{*}$ & $14 / 1$ \\
\hline Li et al. ${ }^{[17]}$ & 48 & 62.4 & LS, Sg1 & $48 / 0$ & 150 & 276 & - & 100 & 72.9 & 9 & $58.3 / 10.4$ \\
\hline Guerra et al. ${ }^{[18]}$ & 59 & 64 & LS, PS & $4 / 78$ & 200 & 210 & 12 & 100 & 92 & 6.7 & $27 / 5$ \\
\hline Goel et al. ${ }^{[19]}$ & 27 & 54 & LS & $0 / 27$ & 200 & 295 & 14.8 & 100 & 100 & 4 & $3.7 / 3.7$ \\
\hline Khan et al. ${ }^{[20]}$ & 61 & 66 & LS & $8 / 53$ & 100 & 240 & 11.5 & 100 & 85.2 & 5 & $37.7 / 11.4$ \\
\hline Efanov et al..$^{[21]}$ & 40 & 45 & PS & $2 / 49$ & $465^{\star}$ & 407 & 0 & 28 & - & 11 & $20 /-$ \\
\hline Choi et al. ${ }^{[22]}$ & 69 & 53 & LS & $64 / 16$ & 170 & 491 & 9.1 & 76.8 & 100 & 8 & $43.5 / 10.6$ \\
\hline Sham et al. ${ }^{[23]}$ & 71 & 54.8 & LS, Sg1 & $17 / 54$ & $495^{\star}$ & $284^{*}$ & 5.7 & 98.6 & - & $3.9^{\star}$ & $14.3 / 4.3$ \\
\hline Fruscione et al. ${ }^{[24]}$ & 57 & 58.1 & LS & $57 / 0$ & 250 & 194 & - & 64.9 & 91.9 & 4 & $28.1 / 25.1$ \\
\hline Lim et al. ${ }^{[25]}$ & 61 & 66 & LS, PS & $9 / 52$ & - & 277 & 0 & 100 & 89 & 9 & $25 / 2$ \\
\hline Beard et al. ${ }^{[26]}$ & 115 & 61 & LS, PS & $17 / 98$ & - & $272^{\star}$ & 5.2 & 93.9 & 73.7 & 5 & $31.3 / 10.4$ \\
\hline Quijano et al. ${ }^{[27]}$ & 21 & 59.3 & LS, PS & $4 / 17$ & - & $262^{*}$ & 4.75 & 65 & - & $12^{*}$ & $19 / 4.7$ \\
\hline Chen et al. ${ }^{[28]}$ & 183 & 60.8 & LS, PS & $92 / 91$ & 249 & 361 & 1.6 & 67.2 & - & 7.5 & $4.4 / 2.1$ \\
\hline Kingham et al. ${ }^{[29]}$ & 64 & 64 & LS & $6 / 65$ & 100 & 163 & 6.3 & 78.2 & - & - & $10.9 / 4.4$ \\
\hline Sucandy et al. ${ }^{[30]}$ & 75 & 64 & LS & $25 / 50$ & 125 & 227 & 0 & 81 & - & 3 & $11 /-$ \\
\hline Wang et al. ${ }^{[31]}$ & 92 & 54.1 & LS & $92 / 0$ & $243^{*}$ & $195.5^{\star}$ & 1 & 66.3 & - & $7.4^{*}$ & $13 / 1.1$ \\
\hline Melstrom et al. ${ }^{[32]}$ & 97 & 62 & LS, PS & $13 / 84$ & $144^{\star}$ & $197^{\star}$ & 9.7 & 85.5 & - & - & $9.7 /-$ \\
\hline Ceccarelli et al. ${ }^{[33]}$ & 70 & 67 & LS, PS & $2 / 89$ & 25 & $115^{\star}$ & 10 & 70 & 94.3 & 3 & $10.1 / 1.4$ \\
\hline Guadagni et al. ${ }^{[34]}$ & 20 & 66 & LS & $0 / 20$ & $250^{*}$ & $198.5^{*}$ & 0 & 20 & 100 & 4.7 & $25 / 0$ \\
\hline
\end{tabular}

Cases: number of patients. Lesions' location: PS: postero-superior segments; LS: laparoscopic segments different from the posterosuperiors; LLS: left-lateral sectionectomy. Major/minor resections: number of major/minor, according to the description of the authors or calculated from the data supplied. EBL: milliliters (median/ ${ }^{*}$ mean). Operative time: minutes (median/ ${ }^{*}$ mean). Conversion rate: percentage of procedures converted to open surgery. Malignant: percentage of malignant lesions. RO: percentage of negative margin status. LoS: days (median/“mean). Overall/major complications: percentage of all complications/major complications. "-": not reported

\section{Surgery related factors}

Among the 29 studies collected, the median value of EBL was $200 \mathrm{~mL}$ (range 25-495) and the median operative time was $260.65 \mathrm{~min}$ (range 107-491). Chong et al. ${ }^{[1]}$ reported results of resections differentiated by difficulty scoring system (DSS). The authors confirmed a correlation between DSS and EBL and operative time. The mean EBL was $274.6 \mathrm{~mL}(146.4 \mathrm{~mL}$ for low difficulty resections and $646.7 \mathrm{~mL}$ for high difficulty resections), while the mean operative time was $259.3 \mathrm{~min}$ (205.9 $\mathrm{min}$ for low difficulty resections and $433.1 \mathrm{~min}$ for high difficulty resections $)^{[35]}$. Daskalaki et al. ${ }^{[12]}$ indicated the specific results of major and minor resections, showing higher EBL and conversion rate, longer operative time and LOS for major resections: mean EBL $354.7 \mathrm{~mL} v$ s. $570 \mathrm{~mL}$, mean operative time $223.2 \mathrm{~min} v s .404 \mathrm{~min}$, conversion rate $2.5 \%$ vs. $17.2 \%$, mean LOS 5.2 days vs. 8.8 days.

Only 10 articles reported data about the use of the Pringle maneuver and the median rate of its application was $23.6 \%$ (range 0-55.6). The agreement in the literature on this topic is limited and some authors considered pedicle clamping unnecessary in most cases during $\mathrm{RS}^{[13-15,36]}$. Otherwise, other authors preferred a routine use of pedicle clamping during major hepatectomies or for difficult resections ${ }^{[2,16-18]}$. 
Conversion to open surgery occurred with a median of 5.45\% (range 0-14.8). Four authors reported that higher conversions rates (greater than 10\%) were related to bleeding, adhesions, technical difficulty, advanced oncological diseases and the requirement of adequate oncologic margins ${ }^{[2,18-20]}$.

\section{Histopathology}

Among the indications for RS of the 29 articles reviewed, malignancies were the $84 \%$ of the cases, in particular the most frequent indication was HCC (40\%), followed by CRLM (21\%), other metastases (14\%), cholangiocarcinoma (CCC) (9\%), GBC (3\%) and other malignancies (13\%). The median tumor size was $33 \mathrm{~mm}$ (range 17.8-73). Efanov et al. ${ }^{[21]}$ emphasized that resections of greater tumors (up to $73 \mathrm{~mm}$ ) should be performed by RS at the end of the surgeon's learning process. The median rate of Ro margin status was 95.5\% (range 72.9-100).

Interestingly, Khan et al. ${ }^{[20]}$ published an international multicenter study, in which they stratified their results for RS by tumor type (3-years overall survival was $90 \%$ for HCC, $65 \%$ for GBC and $49 \%$ for CCC) and reached comparable long-term outcomes, such as overall survival (OS) and disease free survival (DFS), to those of open and laparoscopic liver resections available in literature.

In conclusion, despite the lack of long-term results available in literature, RS is considered feasible and effective in the treatment of malignant diseases.

\section{Short-term postoperative outcomes}

ICU admission rate was described in 6 studies reporting a median frequency of $27.9 \%$ of patients requiring ICU postoperative care (range 0-83.8). Daskalaki et al. ${ }^{[12]}$, even if reporting the $83.8 \%$ of ICU admission after RS, described a reduction in the length of the ICU stay in comparison with open surgery (2.1 days vs. 3.3 days, respectively).

The median rate of overall complications of the 29 reports reviewed was $18.5 \%$ (range 1.7-68.2), with a median rate of major complications (Clavien-Dindo grade 3 or greater) of $4.7 \%$ (range 0-25). Choi et al. ${ }^{[22]}$ reported a greater frequency of overall and major complications in minor resections compared to major hepatectomies ( $46.7 \%$ vs. $42.6 \%$ and $13.3 \%$ vs. 9.3\%, respectively), otherwise Daskalaki et al. ${ }^{[12]}$ described a major rate of overall and major complications in major resections (31\% vs. $15.3 \%$ and $6.8 \%$ vs. $2.5 \%$, respectively).

The median LOS was 5.05 days (range 3-12). In particular, 16 studies reporting an operative time longer than 250 min revealed greater LOS, overall and major complications. Among these 16 articles, the median operative time was $294.2 \mathrm{~min}$ (range 251.5-491) and the corresponding median LOS was 6.4 days (range 3.9-12), overall complications rate was $9 \%$ (range 1-36) and major complications rate was $3.5 \%$ (range $1-12)$.

\section{Hospital costs for RS}

Many studies documented the costs of robotic liver resections, which were higher than laparoscopy, but lower than open surgery. Daskalaki et al. ${ }^{[12]}$ published a retrospective single center comparative study between robotic and open liver surgery, describing higher average costs for open surgery $(\$ 37,518 v s$. $\$ 41,948$ ) including readmissions costs, mainly because of the significant impact of ICU stay, inpatient nursing, and pharmacy costs. Similarly, Sham et al. ${ }^{[23]}$ revealed higher perioperative costs, but significantly lower postoperative and total hospital direct costs for RS ( $\$ 14,754$ vs. $\$ 18,998)$, encouraging the development of RS. 
Table 2. Robotic vs. laparoscopic liver resections

\begin{tabular}{|c|c|c|c|c|c|c|c|c|c|c|c|c|c|c|c|c|}
\hline \multirow[t]{2}{*}{ Authors } & \multicolumn{2}{|c|}{ Cases } & \multicolumn{2}{|c|}{ Location } & \multicolumn{2}{|c|}{ Major/minor } & \multicolumn{2}{|c|}{ EBL } & \multicolumn{2}{|c|}{ Time } & \multicolumn{2}{|c|}{ Conversion } & \multicolumn{2}{|c|}{ LoS } & \multicolumn{2}{|c|}{$\begin{array}{l}\text { Overall/major } \\
\text { complications }\end{array}$} \\
\hline & $\mathbf{R}$ & $\mathbf{L}$ & $\mathbf{R}$ & $\mathbf{L}$ & $\mathbf{R}$ & $\mathbf{L}$ & $\mathbf{R}$ & $\mathbf{L}$ & $\mathbf{R}$ & $\mathbf{L}$ & $\mathbf{R}$ & $\mathbf{L}$ & $\mathbf{R}$ & $\mathbf{L}$ & $\mathbf{R}$ & $\mathbf{L}$ \\
\hline Chong et al. ${ }^{[1]}$ & 91 & 92 & LS, Sg1 & $\begin{array}{l}\text { LS, } \\
\text { Sg1 }\end{array}$ & $19 / 72$ & $4 / 88$ & $275^{\star}$ & $212^{*}$ & $259^{*, \#}$ & $217^{\text {*.\# }}$ & 7.7 & 12 & 4.8 & 4.9 & $9.8 / 3.3$ & $5.4 / 0$ \\
\hline Montalti et al. ${ }^{[2]}$ & 36 & 72 & PS & PS & $0 / 36$ & $0 / 72$ & $415^{\star}$ & $437^{\star}$ & $306^{\star}$ & $295^{\star}$ & 14 & 9.7 & $6^{\star}$ & $4.9^{\star}$ & $19.4 / 11.1$ & $19.4 / 6.9$ \\
\hline Magistri et al..$^{[6]}$ & 22 & 24 & LS, PS & LS, PS & $2 / 20$ & $0 / 24$ & 400 & 328 & $318^{\text {*.\# }}$ & $211^{\text {*.\# }}$ & $0^{\#}$ & $16.7^{\#}$ & 5.1 & 6.2 & $68.1 / 9$ & $100 / 12.5$ \\
\hline Hu et $a l^{[13]}$ & 58 & 54 & LLS & LLS & $0 / 58$ & $0 / 54$ & $80^{\star}$ & $109^{*}$ & $107^{\star}$ & $96^{\star}$ & 0 & 1.9 & 4.3 & 4.4 & $1.7 /-$ & $3.7 /-$ \\
\hline Lee et al. ${ }^{[14]}$ & 70 & 66 & LS & LS & $14 / 70$ & $2 / 66$ & 100 & 100 & $251^{\#}$ & $215^{\#}$ & 5.7 & 12.1 & 5 & 5 & $11.4 /-$ & $4.5 /-$ \\
\hline Lai et al. ${ }^{[16]}$ & 95 & 35 & LS, PS & LS & $27 / 75$ & $1 / 34$ & 335 & 336 & $207^{\star \text {,\# }}$ & $134^{\star, \#}$ & 4 & 5.7 & $7.3^{\star}$ & $7.1^{\star}$ & $14.7 / 1$ & $20 /-$ \\
\hline Efanov et al. ${ }^{[21]}$ & 40 & 91 & LS, PS & LS, PS & $2 / 40$ & $11 / 91$ & 465 & 302 & $407^{\#}$ & $296^{\#}$ & 0 & 0 & $11^{\#}$ & $9^{\#}$ & $20 /-$ & $16.4 /-$ \\
\hline Fruscione et al. ${ }^{[24]}$ & 57 & 116 & LS & LS & $57 / 0$ & $116 / 0$ & 250 & 400 & 194 & 204 & - & - & 4 & 5 & $28 / 7$ & $35.3 / 9.4$ \\
\hline Lim et al..$^{[25]}$ & 55 & 55 & LS, PS & LS, PS & $4 / 51$ & $8 / 47$ & - & - & 254 & 257 & 0 & 0 & 9 & 7 & $21.8 / 1.8$ & $12.7 / 0$ \\
\hline Beard et al. ${ }^{[26]}$ & 115 & 115 & LS, PS & LS, PS & $97 / 18$ & $94 / 21$ & - & - & $272^{\star}$ & $253^{*}$ & $5.2^{\#}$ & $12^{\#}$ & 5 & 4 & $31.3 / 10.4$ & $27.8 / 14.7$ \\
\hline Wang et al..$^{[31]}$ & 92 & 48 & LS & LS & $92 / 0$ & $48 / 0$ & $243^{*, \#}$ & $346^{\star, \#}$ & $195^{\star}$ & $199^{*}$ & $1^{\#}$ & $10.4^{\#}$ & $7.4^{\star}$ & $7^{\star}$ & $13 / 1$ & $10.4 / 0$ \\
\hline Spampinato et al. ${ }^{[36]}$ & 25 & 25 & LS & LS & $25 / 0$ & $25 / 0$ & 250 & 400 & 430 & 360 & 4 & 4 & 8 & 7 & $16 / 4$ & $48 / 12$ \\
\hline Kim et al. ${ }^{[37]}$ & 12 & 31 & LLS & LLS & $0 / 12$ & $0 / 31$ & 225 & 150 & $404^{\#}$ & $246^{\#}$ & - & 1 & 7 & 7 & $-/ 16.6$ & $-/ 9.6$ \\
\hline Packiam et al. ${ }^{[38]}$ & 11 & 18 & LLS & LLS & $0 / 11$ & $0 / 18$ & 30 & 30 & 175 & 188 & 0 & 0 & $4^{\#}$ & $3^{\#}$ & $27.2 / 0$ & 0 \\
\hline Salloum et al. ${ }^{[39]}$ & 14 & 14 & LLS & LLS & $0 / 14$ & $0 / 14$ & $265^{\star}$ & $121^{\star}$ & $203^{\star, \#}$ & $140^{\star \text {,\# }}$ & 14 & 0 & $6^{\star}$ & $6^{\star}$ & $7.1 / 0$ & 7.1/21.4 \\
\hline Croner et al. ${ }^{[40]}$ & 10 & 19 & LS & LS & $0 / 10$ & - & 306 & 356 & 321 & 242 & - & - & 7 & 8 & $10 / 0$ & $15.7 / 5.2$ \\
\hline Ji et al. ${ }^{[41]}$ & 13 & 20 & LS, Sg1 & LS & $9 / 4$ & $4 / 16$ & 280 & 350 & 338 & 130 & 0 & 10 & 6.7 & 5.2 & $7.7 /-$ & $10 /-$ \\
\hline Wu et al. ${ }^{[42]}$ & 52 & 69 & LS & LS & $20 / 52$ & $10 / 69$ & $325^{\circ}$, *, \# & $173^{\circ}$; *\# & $380^{\circ}$ & $227^{\circ \text { :\# }}$ & $5^{\circ}$ & $12^{\circ}$ & $7.9^{\circ}$ & $7.2^{\circ}$ & $5.7 / 0^{\circ}$ & $5.7 /-^{\circ}$ \\
\hline Troisi et al. ${ }^{[43]}$ & 40 & 223 & LS, PS & LS, PS & $0 / 40$ & $82 / 223$ & $330^{\#}$ & $174^{\#}$ & 271 & 262 & 20 & 7.6 & 6.1 & 5.9 & $12.5 / 10$ & $12.5 / 8.9$ \\
\hline Tsung et al. ${ }^{[44]}$ & 57 & 114 & LS & LS & $21 / 36$ & $42 / 72$ & 200 & 100 & $253^{\#}$ & $198^{\#}$ & 7 & 8.8 & 4 & 4 & $19.2 / 1.7$ & $25.4 / 0.8$ \\
\hline Tranchart et al. ${ }^{[45]}$ & 28 & 28 & LS, PS & LS, PS & $0 / 28$ & $0 / 28$ & 200 & 150 & $210^{\#}$ & $176^{\#}$ & 14 & 7.1 & 6 & 5.5 & $17.8 / 10.7$ & $17.8 / 10.7$ \\
\hline Berber et al. ${ }^{[46]}$ & 9 & 23 & LLS & LLS & $0 / 9$ & $0 / 12$ & $136^{\star}$ & $155^{\star}$ & $258^{\star}$ & $234^{\star}$ & 1 & 0 & - & - & $11 /-$ & $17 /-$ \\
\hline Yu et al. ${ }^{[47]}$ & 13 & 17 & LS & LS & $3 / 10$ & $11 / 6$ & 388 & 343 & 291 & 241 & 0 & 0 & $7.8^{*}$ & $9.5^{\star}$ & 0 & $11.7 /-$ \\
\hline Zeng et al. ${ }^{[48]}$ & 3 & 5 & LS & LS & $0 / 3$ & $0 / 5$ & $316^{\star}$ & $290^{*}$ & $370^{*}$ & $249^{\star}$ & 0 & 20 & 3 & 5 & - & - \\
\hline Lin et al. ${ }^{[49]}$ & 25 & 11 & LS & LS & $3 / 25$ & $2 / 11$ & 271 & 295 & 319 & 315 & - & - & 7.5 & 7 & $24 /-$ & $27.2 /-$ \\
\hline
\end{tabular}

In case of PSM, only its data were reported. R: robotic surgery; L: laparoscopic surgery. ${ }^{\circ}$ : referred to the sub-group of HCC. Cases: number of patients. Lesions location: PS:postero-superior segments; LS: laparoscopic segments different from the postero-superiors; LLS: left-lateral sectionectomy. Major/minor resections: number of major/minor, according to the description of the authors or calculated from the data supplied. EBL: milliliters (median/ ${ }^{\star}$ mean). Operative time: minutes (median $/{ }^{*}$ mean). Conversion rate: percentage of procedures converted to open surgery. LoS: days (median $/{ }^{*}$ mean). Overall/major complications: percentage of all complications/major complications. Statistically significant results $(P$-value $<0.05)$ are expressed "\#", if reported in the articles. "-": not reported

\section{Robotic vs. laparoscopic liver surgery}

Twenty-five comparative studies between robotic and laparoscopic liver surgery, including 1,043 cases (range 3-115) and 1,385 cases (range 5-223) respectively, were reviewed [Table 2]. These reports were published from 2010 to 2020. All of them were retrospective and 5 were propensity score matching studies (PSM). In case of PSM only results of the matchings were considered.

\section{Left lateral sectionectomy}

Left lateral sectionectomy (LLS) is currently performed with laparoscopy as a standard of care. Five studies focused on robotic and laparoscopic LLS, including 106 (range 9-58) and 206 (range 18-80) cases, respectively. Most of the articles reported similar perioperative outcomes between laparoscopy and RS. Many authors concluded that laparoscopic LLS remains the gold standard, since RS did not add any significant benefit, but increased the costs ${ }^{[13,37-39]}$. However, Hu et al. ${ }^{[13]}$ established that RS could be the best choice to treat complex cases of LLS (tumor size $>10 \mathrm{~cm}$ in diameter, proximity of the tumor to major vessels, BMI $>30 \mathrm{~kg} / \mathrm{m}^{2}$, combined lymphadenectomy or choledochoscopy, huge left lateral section embedded in splenic fossa), reporting significantly lower EBL than in laparoscopy for these cases (131.9 mL vs. $320.8 \mathrm{~mL}$, respectively). 


\section{Other types of resection}

Among the 25 articles reviewed, the numbers of major and minor robotic resections included were 395 and $694(63.7 \%$ and 36.3\%) respectively, while laparoscopic cases were 460 and 1,002 (68.5\% and 31.5\%), respectively.

Three papers focused on only major hepatectomies (174 robotic vs. 189 laparoscopic cases). Among these, Fruscione et al ${ }^{[24]}$ revealed that robotic technical advantages could improve surgical outcomes in comparison with laparoscopy, reducing postoperative ICU admissions (43.9\% vs. $61.2 \%$ ) and 90-day readmissions ( $7 \%$ vs. $28.5 \%)$, with a similar median complications rate $(28.1 \%$ vs. $35.3 \%)$ and median LOS (4 days $v s .5$ days).

Many authors focused on the ability of RS to overcome laparoscopic drawbacks, particularly simplifying hilar and hepatocaval dissection, suturing and anastomosis, precise vessel dissection or advanced sewing. However, the numbers of complex parenchymal sparing resections involving postero-superior segments or caudate lobe were similar for RS and laparoscopy, 112 (10.7\%) vs. 235 (16.9\%), respectively.

In complex cases many comparative studies demonstrated similar safety, feasibility and postoperative outcomes, but RS was preferred over laparoscopy, especially when several and multiplanar transection lines were necessary, resulting in safe surgical margins and increasing the rate of MIS resections ${ }^{[2,21,26]}$. In the future these advantages could encourage the choice of RS in challenging cases, otherwise not feasible by laparoscopy ${ }^{[40,41]}$.

\section{Surgery related factors}

Considering the 25 articles reviewed, the median EBL for RS and laparoscopy were $261 \mathrm{~mL} v$ s. $290 \mathrm{~mL}$ (range 30-465 vs. 30-457, respectively). Only three studies reported statistically significant differences of this parameter. Wu et al. ${ }^{[42]}$ and Troisi et al. ${ }^{[43]}$ reported greater EBL for RS $(325 \mathrm{~mL} v s .173 \mathrm{~mL}$ and $330 \mathrm{~mL}$ vs. $174 \mathrm{~mL}$, respectively), in contrast with Wang et al. ${ }^{[31]}(243 \mathrm{~mL} v s .346 \mathrm{~mL})$.

Referring to the use of the Pringle maneuver, Montalti et al. ${ }^{[2]}$ reported its significant use during RS compared to in laparoscopy ( $55.6 \%$ vs. $22.2 \%$, respectively) because of the crush technique, leading to a longer inflow occlusion time and greater severity of complications, evaluated by comprehensive complication index (CCI: $34.6 \%$ vs. $18.4 \%$ ). Conversely, Spampinato et al. ${ }^{[36]}$ published a retrospective comparative multi-institutional study, demonstrating that RS allowed for easier management of bleeding during the transection, making the application of the Pringle maneuver less necessary and reporting a significantly higher EBL for laparoscopy compared to RS (400 mL vs. $250 \mathrm{~mL}$, respectively).

The median operative time for RS and laparoscopy was $271 \mathrm{~min}$ (range 107-430) and 227 min (range 96-360), respectively. Ten studies reported statistically significant longer duration with RS compared to laparoscopy, with a mean additional time of $68 \mathrm{~min}$ (range $34-153)^{[1,6,14,16,21,37,39,42,44,45]}$. Spampinato et al. ${ }^{[36]}$ specified that longer robotic operative time could be related to instrument replacement and docking time, which could be reduced by improving the training of the surgical team.

In 18 articles the median operative time was longer than $250 \mathrm{~min}$ for RS and/or laparoscopy. In these studies, although the frequency of minor resections (69.6\% vs. 74.3\%, respectively), EBL ( $293 \mathrm{~mL} v s .292 .5 \mathrm{~mL}$, respectively), LOS (6.7 days vs. 6.2 days, respectively) and overall complications rates ( $16 \% v s .15 .7 \%$, respectively) were similar between RS and laparoscopy, the conversion rate ( $5 \%$ vs. 9.25\%, respectively) was lower for RS. These results suggest that RS could increase MIS approach also in complex cases requiring longer operative time. 
The reported use of hand-port in RS is lower than in laparoscopy. The reason for this observation could be the distance of the first surgeon from the patient and from the operative field that is mainly occupied by the robotic arms. Moreover, the second surgeon at the operative table could not have enough surgical skills to manage unexpected events. This statement could explain also the lower rate of conversion to hybrid robotic procedures in case of unexpected events. However, many studies reported an easier robotic management of adhesions and major intraoperative complications as bleeding than in laparoscopy, that could explain the lower rate of conversion to open surgery for RS.

The median rates of conversion for RS and laparoscopy were $4 \% v s .7 .35 \%$ (both ranges $0-20$ ). Among the 25 comparative articles reviewed, 4 papers reported a statistically significant higher conversion rate for laparoscopy in comparison with $\mathrm{RS}^{[6,26,31]}$, while the other authors did not reach statistically significant results for this variable. Only Troisi et al. ${ }^{[43]}$ found a higher conversion rate for RS compared to laparoscopic surgery $(20 \%$ vs. $7.6 \%, P=0.034)$ but, considering only resections of postero-superior segments, they showed that RS provided a lower conversion rate $(20 \% v s .35 .3 \%, P=0.38)$.

\section{Postoperative outcomes}

The median rates of overall robotic and laparoscopic complications were $17.7 \%$ vs. 37.6\% (ranges 5.7-68.1 vs. 27.2-48), respectively. The median rates of major complications were $2.5 \%$ vs. $8.9 \%$ (ranges 0-16.6 vs. $0-21.4)$, respectively.

The median LOS for RS and laparoscopy were 6 days vs. 5.95 days (ranges 3-11 vs. 3-9.5). Only Efanov et al. ${ }^{[21]}$ and Packiam et al. ${ }^{[38]}$ reported a statistically significant longer hospital stay for RS, mainly caused by postoperative complications and ICU stay.

\section{Comparison of costs}

Many authors confirmed the major costs of robotic resections, although the annual service fees could be cushioned by the utilization of the robot in other surgical specialties at the same institution. Kim et al. ${ }^{[37]}$ observed that robotic LLS showed higher costs $(\$ 8,183$ vs. $\$ 5,190)$ and longer operative time. Salloum et al. ${ }^{[39]}$ suggested that robotic LLS did not add additional advantages in comparison with laparoscopic outcomes. Furthermore, while perioperative costs were higher in the robotic group, total costs were similar in comparison with laparoscopy $(€ 5,522 v s$. $€ 6,035)$. Berber et al. ${ }^{[46]}$ calculated a general addition of $\$ 500$ per case for the robotic equipment. Ji et al. ${ }^{[41]}$ considered RS not routinely applicable, since its higher costs in comparison with laparoscopy $(\$ 12,046 v$ s. $\$ 7,618)$. Packiam et al. ${ }^{[38]}$ performed a cost analysis differentiating direct and indirect costs of RS. Only robotic indirect costs were significantly higher, adding $\$ 1,423$ per case $(\$ 6,553$ vs. $\$ 4,408)$. However, Yu et al. ${ }^{[47]}$ concluded that RS could really increase in near future, overcoming the drawbacks represented by the major costs $(\$ 11,475 v s . \$ 6,762)$ and the absence of transection tools equivalent to those available in laparoscopy.

\section{Robotic surgery in specific malignant diseases}

The majority of the publications in the literature report cumulative results, without differentiation between benign and malignant diseases. However, in future probably more specific analyses of RS outcomes for each of the most relevant hepatobiliary malignancies could help in the definition of the standard of care for each one.

\section{Hepatocellular carcinoma}

Robotic resections for HCC are feasible, safe, and demonstrated adequate oncologic outcomes. Six retrospective papers, including 294 patients, analyzed the results of RS for HCC [Table 3].

In this field the superiority of robotic MIS over open surgery was confirmed by Chen et al. ${ }^{[28]}$ by a PSM study. Even in challenging major resections, robotic approach showed longer operative time, but shorter 
Table 3. Robotic surgery for hepatocellular carcinoma

\begin{tabular}{|c|c|c|c|c|c|c|c|c|c|c|c|c|}
\hline Authors & Cases & Age & Location & $\begin{array}{c}\text { Major/ } \\
\text { minor }\end{array}$ & EBL & Time & Conversion & Cirrhosis & RO & LoS & $\begin{array}{l}\text { Overall/major } \\
\text { complications }\end{array}$ & DFS/OS \\
\hline Magistri et al. ${ }^{[6]}$ & 22 & 60.8 & LS, PS & $2 / 20$ & 400 & $318^{\star}$ & 0 & 68 & 95 & 5.1 & $68 / 9$ & - \\
\hline Lai et al. ${ }^{[16]}$ & 95 & 62.1 & LS, PS & $27 / 75$ & 335 & 207 & 4 & 84 & 96 & $7.3^{\star}$ & $14 / 1$ & 5-year: $42 / 65$ \\
\hline Lim et al. ${ }^{[25]}$ & 42 & - & - & - & - & - & 0 & - & 97 & - & - & 3-year: 64/98 \\
\hline Chen et al. ${ }^{[28]}$ & 81 & - & - & $34 / 47$ & 282 & 343 & - & 46 & 97 & 7.5 & $5 / 0$ & 3-year: 72/93 \\
\hline Wu et al. ${ }^{[42]}$ & 38 & 60.9 & LS & - & - & 380 & 5 & - & - & 7.9 & $8 /-$ & - \\
\hline Han et al. ${ }^{[50]}$ & 16 & 54.5 & LS & $10 / 16$ & 389 & 285 & 0 & 53 & 100 & 8.4 & - & - \\
\hline
\end{tabular}

Cases: number of patients. Lesions' location: PS: postero-superior segments; LS: laparoscopic segments different from the posterosuperiors. OS: overall survival; DFS: disease free survival. Major/minor resections: number of major/minor, according to the description of the authors or calculated from the data supplied. EBL: milliliters (median $/{ }^{*}$ mean). Operative time: minutes (median $/{ }^{*}$ mean). Conversion rate: percentage of procedures converted to open surgery. RO: percentage of negative margin status. LoS: days (median/ ${ }^{\star}$ mean). Overall/ major complications: percentage of all complications/major complications. DFS and OS: percentage at 3-/5-year. "-": not reported

LOS, improved patients' pain control, not compromising oncologic outcomes and reaching comparable 3-years DFS (72.2\% vs. 58.0\%) and 3-years OS (92.6\% vs. 93.7\%). Magistri et al. ${ }^{[6]}$ and Lai et al. ${ }^{[16]}$ reported less minor robotic postoperative complications, such as pleural effusion, thanks to gentler manipulation of the diaphragm, especially in the case of lesions located in postero-superior segments. In addition, RS allowed lower rates of conversion, a greater number of resections involving the postero-superior segments, and resections of slightly larger tumors, that could explain the higher rate of major hepatectomies. Lai et al. ${ }^{[16]}$ did not find significant differences between robotic and laparoscopic oncologic outcomes (5-years OS: $65 \%$ vs. $48 \%$, respectively), morbidity and mortality. The authors concluded that robotic MIS was a valid alternative treatment for HCC in selected patients and in the hands of surgeons expert in laparoscopic and robotic liver surgery, following the principles of open liver surgery.

Likewise, Han et al. ${ }^{[50]}$ revealed the safety and feasibility of the robotic approach to complex procedures and anatomical liver resections, and thus the superiority of minimally invasive liver surgery in terms of EBL, complication rate, LOS and risk of ascites, maintaining DFS and OS similar to open surgery.

\section{Gallbladder cancer}

RS seems particularly advantageous in the treatment of GBC, overcoming the difficulties related to the laparoscopic approach. Focusing on this field, 4 articles including 51 patients were reviewed [Table 4]. Zeng et al ${ }^{[48]}$ demonstrated safety and feasibility of both robotic and laparoscopic surgery. Otherwise, Goel et al. ${ }^{[19]}$ and Byun et al..$^{[51]}$ compared results of robotic and open radical cholecystectomy. They achieved similar results between the two approaches, reaching no significant differences in operative time, EBL and number of retrieved lymph nodes, with a reduction of LOS. Likewise, Shen et al. ${ }^{[52]}$ confirmed the feasibility of a complete robotic lymphadenectomy of the hepatic artery, the celiac axis, the hepatoduodenal ligament and retropancreatic nodes, in contrast with laparoscopy. In addition, robotic approach could reduce the risk of major iatrogenic injuries and major bleeding could be more easily managed ${ }^{[51,52]}$.

In conclusion, in selected cases RS for GBC is considered safe, feasible and effective, even during the initial learning curve, allowing sufficient lymph node dissection and enhancing recovery ${ }^{[1,51,52]}$.

\section{Hilar cholangiocarcinoma}

Even if the advantages of the robotic technique for procedures that require extreme precision and microanastomosis are clear, the scientific literature is lacking in reports about robotic treatment of this disease. Probably further implementation in surgeon expertise and robotic tools are necessary to reach encouraging results that could increase its use. Two articles were selected and their data tabulated [Table 5].

Li et al. ${ }^{[17]}$ highlighted the feasibility of 48 robotic resections for Bismuth-Corlette type I, II or III hilar cholangiocarcinoma. The authors considered RS a valid alternative to open surgery in selected cases, 
Table 4. Robotic surgery for gallbladder cancer

\begin{tabular}{|c|c|c|c|c|c|c|c|c|c|c|}
\hline Authors & Cases & Age & EBL & Time & Conversion & Lymph nodes & Extension & RO & LoS & $\begin{array}{l}\text { Overall/major } \\
\text { complications }\end{array}$ \\
\hline Goel et al. ${ }^{[19]}$ & 27 & 54 & 200 & 295 & 14.8 & $10 /-$ & pT2-3 & 100 & 4 & $3.7 / 3.7$ \\
\hline Zeng et al..$^{[48]}$ & 3 & - & $316^{\star}$ & $370^{\star}$ & 0 & $6.3 /-$ & pT2-3 & - & 3 & - \\
\hline Byun et al..$^{[51]}$ & 16 & 64.3 & 295 & $198.3^{*}$ & - & $7.2 / 3$ & - & 100 & 7 & $6.3 / 6.3$ \\
\hline Shen et al. ${ }^{[52]}$ & 5 & 57.4 & $210^{*}$ & $200^{\star}$ & 0 & $9 / 1,3$ & - & - & $7.4^{\star}$ & 0 \\
\hline
\end{tabular}

Cases: number of patients. Major/minor resections: number of major/minor, according to the description of the authors or calculated from the data supplied. EBL: milliliters (median/ ${ }^{\star}$ mean). Operative time: minutes (median/ ${ }^{\star}$ mean). Conversion rate: percentage of procedures converted to open surgery. Lymph nodes: mean number of nodes obtained/mean number of positive nodes. RO: percentage of negative margin status. LoS: days (median/ ${ }^{\star}$ mean). Overall/major complications: percentage of all complications/major complications. "-": not reported

Table 5. Robotic surgery for hilar cholangiocarcinoma

\begin{tabular}{|c|c|c|c|c|c|c|c|c|c|c|c|}
\hline Authors & Cases & Age & $\begin{array}{c}\text { Pre-op. } \\
\text { procedures }\end{array}$ & $\begin{array}{c}\text { Type of } \\
\text { resection }\end{array}$ & EBL & $\begin{array}{c}\text { Operative } \\
\text { time }\end{array}$ & Conversion & RO & LoS & $\begin{array}{l}\text { Overall/major } \\
\text { complications }\end{array}$ & Biliary leak \\
\hline Li et al. ${ }^{[17]}$ & 48 & 62.4 & PTBD 41.7 & $\mathrm{RH} / \mathrm{LH}+\mathrm{Sg} 1$ & 150 & 276 & - & 72.9 & 9 & $58.3 / 10.4$ & 4.2 \\
\hline Xu et al. ${ }^{[53]}$ & 10 & 54 & $\begin{array}{l}\text { PVE 10, } \\
\text { PTBD } 60\end{array}$ & $\begin{array}{l}\mathrm{LH} / \mathrm{RH}+\mathrm{Sg} 1(9) \\
\mathrm{ERH}(1)\end{array}$ & 1360 & 703 & 0 & - & 16 & $90 / 30$ & 40 \\
\hline
\end{tabular}

Cases: number of patients. PTBD: percentage of percutaneous trans-hepatic biliary drainage; PVE: percentage of portal vein embolization; RH/LH: right/left hepatectomy; ERH: number of extended right hepatectomies; EBL: milliliters (median/ ${ }^{\star}$ mean). Operative time: minutes (median $/{ }^{*}$ mean). Conversion rate: percentage of procedures converted to open surgery. RO: percentage of negative margin status. LoS: days (median/ ${ }^{\star}$ mean). Overall/major complications: percentage of all complications/major complications. Biliary leak: percentage. " ${ }^{\prime}$ : not reported

allowing lymphadenectomy of groups 7, 8, 9, 12 and 13. However, they did not report details about the extension of hepatectomies for each tumor stage and the rates of conversion to open surgery. Conversely, $\mathrm{Xu}$ et al. ${ }^{[53]}$ evaluated their results for 10 patients of fully robotic-assisted radical resection for hCCC. The authors demonstrated that this procedure is technically achievable in selected patients by expert surgeons, but without superior results to open surgery. In fact, they observed technical limitations in robotic liver mobilization and exposure, longer operative time and massive EBL, consequently increased morbidity, higher costs and poor long-term outcomes with greater rate of peritoneum implantation and multisite metastases.

\section{Colorectal liver metastases}

Many hepatobiliary surgeons encouraged the robotic approach to CRLM, achieving good surgical and oncological outcomes. Seven articles were reviewed, including 242 patients [Table 6]. Beard et al. ${ }^{[26]}$ focused their PSM on RS for CRLM and considered it feasible and safe, being perioperative and long-term oncologic outcomes largely comparable to laparoscopy.

Araujo et al. ${ }^{[54]}$ and Troisi et al. ${ }^{[43]}$ demonstrated feasibility of non-anatomical robotic resections of lesions located in postero-superior segments, simplifying parenchymal sparing resections, not affecting the oncologic outcomes, reducing the necessity of major hepatectomies and overcoming laparoscopic drawbacks.

Fifty-four simultaneous resections of the primary tumor and liver metastases were included. In these cases, RS added additional safety and effectiveness in the management of multiple metastases, improving shortterm outcomes such as EBL, bowel function return time and LOS, with the exception of operative time, reaching excellent Ro resection rates ${ }^{[34,55]}$. Even in selected cases requiring major liver resections, robotic surgery gained acceptable morbidity ${ }^{[56]}$. In addition, it is worth considering that robotic total mesorectal excision demonstrated better preservation of urinary and sexual functions, low conversion rates and favorable morbidity ${ }^{[49]}$. 
Table 6. Robotic surgery for colorectal liver metastases

\begin{tabular}{|c|c|c|c|c|c|c|c|c|c|c|c|c|}
\hline First Author & Cases & Age & Location & $\begin{array}{l}\text { Major/ } \\
\text { minor }\end{array}$ & Simultaneous & EBL & Time & Conversion & RO & LoS & $\begin{array}{l}\text { Overall/major } \\
\text { complications }\end{array}$ & DFS/OS \\
\hline Guerra et al. ${ }^{[18]}$ & 59 & 64 & LS, PS & $4 / 78$ & 4 & 200 & 210 & 12 & 92 & 6.7 & $27 / 5$ & $\begin{array}{l}\text { 3-year: } \\
41.9 / 66.1\end{array}$ \\
\hline Beard et al. ${ }^{[26]}$ & 115 & 61 & LS, PS & $97 / 18$ & - & - & $272^{\star}$ & 5.2 & 73.7 & 5 & $31.3 / 10.4$ & $\begin{array}{l}\text { 5-year: } \\
38 / 61\end{array}$ \\
\hline Guadagni et al. ${ }^{[34]}$ & 20 & 66 & LS & $0 / 20$ & 3 & $250^{*}$ & $198^{\star}$ & 0 & 100 & 4.7 & $25 / 0$ & $\begin{array}{l}\text { 3-year: } \\
35.8 /-\end{array}$ \\
\hline Lin et al. ${ }^{[49]}$ & 25 & 58.5 & LS & $3 / 22$ & 25 & 271 & 319 & - & 100 & 7.5 & $24 /-$ & - \\
\hline Araujo et al. ${ }^{[54]}$ & 5 & 59 & PS & $0 / 5$ & - & $160^{\star}$ & $294^{\star}$ & 0 & 100 & 4 & $20 / 0$ & - \\
\hline Dwyer et al. ${ }^{[55]}$ & 6 & 59.3 & - & $0 / 6$ & 6 & 316 & 401 & 0 & 100 & 4.5 & $33.3 /-$ & - \\
\hline Navarro et al. ${ }^{[56]}$ & 12 & 59 & LS, Sg1 & $4 / 8$ & 16 & 274 & 449 & 0 & 100 & 12 & $41 / 16.6$ & - \\
\hline
\end{tabular}

Cases: number of patients. Lesions' location: PS: postero-superior segments; LS: laparoscopic segments different from the posterosuperiors. Major/minor resections: number of major/minor, according to the description of the Authors or calculated from the data supplied. Simultaneous: resection of the primary and secondary tumors simultaneously. EBL: milliliters (median/ ${ }^{\star}$ mean). Operative time: minutes (median/*mean). Conversion rate: percentage of procedures converted to open surgery. RO: percentage of negative margin status. LoS: days (median/*mean). Overall/major complications: percentage of all complications/major complications. DFS and OS: percentage at 3-/5-year. "-": not reported

In conclusion, these outcomes could support the use of RS, despite the high operative time and costs.

\section{CONCLUSION}

In the field of hepatobiliary surgery, use of the robotic approach is promising, but not standardized yet. International and multicenter studies are limited, only few publications reported long-term outcomes and no randomized trials are available in literature. In the current literature many authors attempted to reach definitive conclusions about the use of RS publishing many reviews/meta-analyses. In general, almost all of these studies found RS as safe and effective with acceptable morbidity in the treatment of liver malignancies as for laparoscopy ${ }^{[7,57-59]}$. Furthermore many authors agreed with the necessity of specific training in RS, the high costs and the usefulness of RS in complex cases, such as cirrhotic patients and in complex surgical procedures including microsuturing, biliary dissection, and bilio-enteric anastomosis ${ }^{[59,60]}$. However many results of RS are still discordant, mainly in short-term outcomes, and no studies reported definitive indications and contraindications of RS because of the lack of randomized control trials. The comparison among open, laparoscopic, and robotic liver resections demonstrated that the robotic approach achieved similar results to other MIS techniques, enhancing patients' recovery after surgery.

The majority of the studies reported single center initial experiences and considered the robotic learning curve shorter than the laparoscopic one, especially for surgeons with advanced skills in open liver surgery $^{[21,22,29,33]}$. Efanov et al. ${ }^{[21]}$ established that 8-10 robotic procedures can be adequate to significantly increase the surgeons' experience and the ability to perform difficult procedures.

Choi et al. ${ }^{[22]}$ reported results from single center and single surgeon's activity, emphasizing the usefulness of high experience on open liver surgery to approach the robotic resections, making safe and feasible all types of anatomic liver resections, even more complex ones, such as staged hepatectomy and living donor right hepatectomy. Ceccarelli et al. ${ }^{[33]}$, describing their robotic learning curve program organized in a network between high and low volume centers, demonstrated that this strategy provides a proper standard of care without the need of reaching referral centers for the patients, even in particularly complex cases. In addition, this protocol can improve surgical skills, shortening the learning curve.

Interestingly, Lai et al. ${ }^{[61]}$ reviewed the learning curves of robotic and laparoscopic hepatectomy and concluded that a qualified robotic surgeon should have great knowledge of liver anatomy, enough experience in open liver surgery and in the management of its major complications and a good training in both laparoscopic and robotic surgery. 
In conclusion, in the era of MIS in which surgical innovations are increasing, even if the younger surgeons are more confident with MIS, both open and laparoscopic surgical experiences are necessary in order to shorten the learning curve of robotic liver surgery and all the surgeons should receive specific training for RS.

Even though robotic liver surgery allows attainment of excellent oncologic results with adequate Ro margins, long-term outcomes are still lacking, probably because of the recent introduction of this technique.

Regarding the type of liver resection, robotic LLS is considered inappropriate in comparison with the laparoscopic one, which is actually the standard of care. In fact, while perioperative outcomes are similar, costs are markedly higher for RS. On the contrary, complex cases could take advantages from RS, thus increasing the rate of MIS.

Even for other type of resections, the results available in the current literature encourage the use of robotic surgery in complex cases, for example for lesions located in postero-superior segments. Furthermore, many studies reported easier management of major intraoperative complications, such as bleeding, that could explain the lower rate of conversion compared with laparoscopy.

Among the comparative studies between MIS techniques, many of them reported a greater number of major resections for robotic surgery. Some authors explained these results with the reduced difficulty of robotic major hepatectomies in comparison with laparoscopy, allowing a potential increase of MIS in more complex cases $^{[24,40,41]}$.

One of the most relevant drawbacks of robotic surgery remain higher costs. Almost all the comparative studies confirmed robotic perioperative higher costs with reduced postoperative ones ${ }^{[23]}$. Even for this reason, many authors encouraged the use of RS only in complex cases.

It is possible that the increasing spread of robotic surgery and the introduction of new robotic platforms with industry competition could lead to a consistent reduction of these costs.

Regarding the application of the robotic approach in specific diseases, RS for HCC and liver metastases achieved good results, allowing parenchymal sparing resections, even in difficult locations.

Furthermore, the robotic approach to biliary tumors seems to be the most promising application of robotic surgery, because of the need for extensive lymph node dissection and of bilio-enteric anastomoses. Currently, there are discordant opinions regarding hCCC, whereas robotic surgery for GBC can add relevant benefits, increasing the rate of MIS without compromising oncologic results.

In conclusion, different techniques should be tailored to each patient, applying MIS when possible, particularly in cirrhotic patients and in the context of liver transplantation ${ }^{[15,34]}$. Thus, in a modern hepatobiliary center, both open surgery and MIS should be provided, including the robotic technique, in order to safely deal with different liver diseases requiring complex procedures ${ }^{[6]}$.

In the future the technological innovation could lead to more complete, less expensive and smaller robotic systems with additional devices or software for RS, that could really change the actual scenario of MIS overcoming many of the drawbacks of RS. For example, it could become possible to approach the operation without the second surgeon, the equipment could be smaller, the docking could be easier and quicker and costs reduced thanks to the market competition. 
Although a lot of hepatobiliary centers worldwide are already experienced in robotic liver surgery, more and larger studies are necessary to define its real benefits compared with laparoscopy, in order to standardize patient selection criteria and its use.

\section{DECLARATIONS}

\section{Authors' contributions}

Designed and performed the research: Ruzzenente A, Alaimo L

Collected the data: Conci S, Bagante F

Analyzed the data: Campagnaro T, Pedrazzani C

Supervised the work: Ruzzenente A, Guglielmi A

Wrote and finally approved the manuscript: Ruzzenente A, Alaimo L, Conci S, Bagante F, Campagnaro T, Pedrazzani C, Guglielmi A

\section{Availability of data and materials}

A search of the current literature on robotic liver surgery was conducted in PubMed, Medline, PMC and Google Scholar databases.

\section{Financial support and sponsorship}

None.

\section{Conflicts of interest}

All authors declared that there are no conflicts of interest.

\section{Ethical approval and consent to participate}

Not applicable.

\section{Consent for publication}

Not applicable.

\section{Copyright}

(c) The Author(s) 2020.

\section{REFERENCES}

1. Chong CCN, Lok HT, Fung AKY, et al. Robotic versus laparoscopic hepatectomy: application of the difficulty scoring system. Surg Endosc 2020;34:2000-6.

2. Montalti R, Scuderi V, Patriti A, Vivarelli M, Troisi RI. Robotic versus laparoscopic resections of posterosuperior segments of the liver: a propensity score-matched comparison. Surg Endosc 2016;30:1004-13.

3. Lafaro KJ, Stewart C, Fong A, Fong Y. Robotic liver resection. Surg Clin North Am 2020;100:265-81.

4. Marino MV, Podda M, Fernandez CC, Ruiz MG, Fleitas MG. The application of indocyanine green-fluorescence imaging during roboticassisted liver resection for malignant tumors: a single-arm feasibility cohort study. HPB (Oxford) 2020;22:422-31.

5. Pesi B, Moraldi L, Guerra F, et al. Surgical and oncological outcomes after ultrasound-guided robotic liver resections for malignant tumor. Analysis of a prospective database. Int J Med Robot 2019;15:e2002.

6. Magistri P, Tarantino G, Guidetti C, et al. Laparoscopic versus robotic surgery for hepatocellular carcinoma: the first 46 consecutive cases. J Surg Res 2017;217:92-9.

7. Fahrner R, Rauchfuß F, Bauschke A, Kissler H, Settmacher U, Zanow J. Robotic hepatic surgery in malignancy: review of the current literature. J Robot Surg 2019;13:533-8.

8. Abu Hilal M, Aldrighetti L, Dagher I, et al. The southampton consensus guidelines for laparoscopic liver surgery: from indication to implementation. Ann Surg 2018;268:11-8.

9. Strasberg S, Belghiti J, Clavien P, et al. The Brisbane 2000 terminology of liver anatomy and resections. HPB 2000;2:333-9.

10. Dindo D, Demartines N, Clavien PA. Classification of surgical complications: a new proposal with evaluation in a cohort of 6336 patients and results of a survey. Ann Surg 2004;240:205-13.

11. Nota CL, Woo Y, Raoof M, et al. Robotic versus open minor liver resections of the posterosuperior segments: a multinational, propensity 
score-matched study. Ann Surg Oncol 2019;26:583-90.

12. Daskalaki D, Gonzalez-Heredia R, Brown M, et al. Financial impact of the robotic approach in liver surgery: a comparative study of clinical outcomes and costs between the robotic and open technique in a single institution. $J$ Laparoendosc Adv Surg Tech A 2017;27:375-82.

13. Hu M, Liu Y, Li C, et al. Robotic versus laparoscopic liver resection in complex cases of left lateral sectionectomy. Int J Surg 2019;67:54-60.

14. Lee KF, Cheung YS, Chong CC, Wong J, Fong AK, Lai PB. Laparoscopic and robotic hepatectomy: experience from a single centre. ANZ J Surg 2016;86:122-6.

15. Felli E, Santoro R, Colasanti M, Vennarecci G, Lepiane P, Ettorre GM. Robotic liver surgery: preliminary experience in a tertiary hepatobiliary unit. Updates Surg 2015;67:27-32.

16. Lai EC, Tang CN. Long-term survival analysis of robotic versus conventional laparoscopic hepatectomy for hepatocellular carcinoma: a comparative study. Surg Laparosc Endosc Percutan Tech 2016;26:162-6.

17. Li J, Tan X, Zhang X, et al. Robotic radical surgery for hilar cholangiocarcinoma: a single-centre case series. Int J Med Robot 2020;16:e2076.

18. Guerra F, Guadagni S, Pesi B, et al. Outcomes of robotic liver resections for colorectal liver metastases. A multi-institutional analysis of minimally invasive ultrasound-guided robotic surgery. Surg Oncol 2019;28:14-8.

19. Goel M, Khobragade K, Patkar S, Kanetkar A, Kurunkar S. Robotic surgery for gallbladder cancer: operative technique and early outcomes. J Surg Oncol 2019;119:958-63.

20. Khan S, Beard RE, Kingham PT, et al. Long-term oncologic outcomes following robotic liver resections for primary hepatobiliary malignancies: a multicenter study. Ann Surg Oncol 2018;25:2652-60.

21. Efanov M, Alikhanov R, Tsvirkun V, et al. Comparative analysis of learning curve in complex robot-assisted and laparoscopic liver resection. HPB (Oxford) 2017;19:818-24.

22. Choi GH, Chong JU, Han DH, Choi JS, Lee WJ. Robotic hepatectomy: the Korean experience and perspective. Hepatobiliary Surg Nutr 2017;6:230-8.

23. Sham JG, Richards MK, Seo YD, Pillarisetty VG, Yeung RS, Park JO. Efficacy and cost of robotic hepatectomy: is the robot costprohibitive? J Robot Surg 2016;10:307-13.

24. Fruscione M, Pickens R, Baker EH, et al. Robotic-assisted versus laparoscopic major liver resection: analysis of outcomes from a single center. HPB (Oxford) 2019;21:906-11.

25. Lim C, Salloum C, Tudisco A, et al. Short- and long-term outcomes after robotic and laparoscopic liver resection for malignancies: a propensity score-matched study. World J Surg 2019;43:1594-603.

26. Beard RE, Khan S, Troisi RI, et al. Long-term and oncologic outcomes of robotic versus laparoscopic liver resection for metastatic colorectal cancer: a multicenter, propensity score matching analysis. World J Surg 2020;44:887-95.

27. Quijano Y, Vicente E, Ielpo B, et al. Hepatobilio-pancreatic robotic surgery: initial experience from a single center institute. J Robot Surg 2017;11:355-65

28. Chen PD, Wu CY, Hu RH, et al. Robotic versus open hepatectomy for hepatocellular carcinoma: a matched comparison. Ann Surg Oncol 2017;24:1021-8.

29. Kingham TP, Leung U, Kuk D, et al. Robotic liver resection: a case-matched comparison. World J Surg 2016;40:1422-8.

30. Sucandy I, Giovannetti A, Spence J, Ross S, Rosemurgy A. Does preoperative MELD score affect outcomes following robotic hepatectomy for liver tumors? J Robot Surg 2020;14:725-31.

31. Wang ZZ, Tang WB, Hu MG, et al. Robotic vs laparoscopic hemihepatectomy: a comparative study from a single center. $J$ Surg Oncol 2019;120:646-53.

32. Melstrom LG, Warner SG, Woo Y, et al. Selecting incision-dominant cases for robotic liver resection: towards outpatient hepatectomy with rapid recovery. Hepatobiliary Surg Nutr 2018;7:77-84.

33. Ceccarelli G, Andolfi E, Fontani A, Calise F, Rocca A, Giuliani A. Robot-assisted liver surgery in a general surgery unit with a "Referral Centre Hub\&Spoke Learning Program". Early outcomes after our first 70 consecutive patients. Minerva Chir 2018;73:460-8.

34. Guadagni S, Furbetta N, Di Franco G, et al. Robotic-assisted surgery for colorectal liver metastasis: a single-centre experience. $J$ Minim Access Surg 2019;16:160-5.

35. Ban D, Tanabe M, Ito H, et al. A novel difficulty scoring system for laparoscopic liver resection. $J$ Hepatobiliary Pancreat Sci 2014;21:745-53.

36. Spampinato MG, Coratti A, Bianco L, et al. Perioperative outcomes of laparoscopic and robot-assisted major hepatectomies: an Italian multi-institutional comparative study. Surg Endosc 2014;28:2973-9.

37. Kim JK, Park JS, Han DH, et al. Robotic versus laparoscopic left lateral sectionectomy of liver. Surg Endosc 2016;30:4756-64.

38. Packiam V, Bartlett DL, Tohme S, et al. Minimally invasive liver resection: robotic versus laparoscopic left lateral sectionectomy. $J$ Gastrointest Surg 2012;16:2233-8.

39. Salloum C, Lim C, Lahat E, et al. Robotic-assisted versus laparoscopic left lateral sectionectomy: analysis of surgical outcomes and costs by a propensity score matched cohort study. World J Surg 2017;41:516-24.

40. Croner RS, Perrakis A, Hohenberger W, Brunner M. Robotic liver surgery for minor hepatic resections: a comparison with laparoscopic and open standard procedures. Langenbecks Arch Surg 2016;401:707-14.

41. Ji WB, Wang HG, Zhao ZM, Duan WD, Lu F, Dong JH. Robotic-assisted laparoscopic anatomic hepatectomy in China: initial experience. Ann Surg 2011;253:342-8.

42. Wu YM, Hu RH, Lai HS, Lee PH. Robotic-assisted minimally invasive liver resection. Asian J Surg 2014;37:53-7. 
43. Troisi RI, Patriti A, Montalti R, Casciola L. Robot assistance in liver surgery: a real advantage over a fully laparoscopic approach? Results of a comparative bi-institutional analysis. Int J Med Robot 2013;9:160-6.

44. Tsung A, Geller DA, Sukato DC, et al. Robotic versus laparoscopic hepatectomy: a matched comparison. Ann Surg 2014;259:549-55.

45. Tranchart H, Ceribelli C, Ferretti S, Dagher I, Patriti A. Traditional versus robot-assisted full laparoscopic liver resection: a matched-pair comparative study. World J Surg 2014;38:2904-9.

46. Berber E, Akyildiz HY, Aucejo F, Gunasekaran G, Chalikonda S, Fung J. Robotic versus laparoscopic resection of liver tumours. $H P B$ (Oxford) 2010;12:583-6.

47. Yu YD, Kim KH, Jung DH, et al. Robotic versus laparoscopic liver resection: a comparative study from a single center. Langenbecks Arch Surg 2014;399:1039-45.

48. Zeng G, Teo NZ, Goh BKP. Short-term outcomes of minimally invasive surgery for patients presenting with suspected gallbladder cancer: Report of 8 cases. J Minim Access Surg 2018;15:109-14.

49. Lin Q, Ye Q, Zhu D, et al. Comparison of minimally invasive and open colorectal resections for patients undergoing simultaneous R0 resection for liver metastases: a propensity score analysis. Int J Colorectal Dis 2015;30:385-95.

50. Han DH, Choi SH, Park EJ, Kang DR, Choi GH, Choi JS. Surgical outcomes after laparoscopic or robotic liver resection in hepatocellular carcinoma: a propensity-score matched analysis with conventional open liver resection. Int J Med Robot 2016;12:735-42.

51. Byun Y, Choi YJ, Kang JS, et al. Robotic extended cholecystectomy in gallbladder cancer. Surg Endosc 2020;34:3256-61.

52. Shen BY, Zhan Q, Deng XX, et al. Radical resection of gallbladder cancer: could it be robotic? Surg Endosc 2012;26:3245-50.

53. Xu Y, Wang H, Ji W, et al. Robotic radical resection for hilar cholangiocarcinoma: perioperative and long-term outcomes of an initial series. Surg Endosc 2016;30:3060-70.

54. Araujo RLC, Sanctis MA, Barroti LC, Coelho TRV. Robotic approach as a valid strategy to improve the access to posterosuperior hepatic segments-case series and review of literature. J Surg Oncol 2020;121:873-80.

55. Dwyer RH, Scheidt MJ, Marshall JS, Tsoraides SS. Safety and efficacy of synchronous robotic surgery for colorectal cancer with liver metastases. J Robot Surg 2018;12:603-6.

56. Navarro J, Rho SY, Kang I, Choi GH, Min BS. Robotic simultaneous resection for colorectal liver metastasis: feasibility for all types of liver resection. Langenbecks Arch Surg 2019;404:895-908.

57. Tsilimigras DI, Moris D, Vagios S, Merath K, Pawlik TM. Safety and oncologic outcomes of robotic liver resections: a systematic review. J Surg Oncol 2018;117:1517-30.

58. Qiu J, Chen S, Chengyou D. A systematic review of robotic-assisted liver resection and meta-analysis of robotic versus laparoscopic hepatectomy for hepatic neoplasms. Surg Endosc 2016;30:862-75.

59. Ocuin LM, Tsung A. Robotic liver resection for malignancy: current status, oncologic outcomes, comparison to laparoscopy, and future applications. J Surg Oncol 2015;112:295-301.

60. Peters BS, Armijo PR, Krause C, Choudhury SA, Oleynikov D. Review of emerging surgical robotic technology. Surg Endosc 2018;32:1636-55.

61. Lai ECH, Tang CN. Training robotic hepatectomy: the Hong Kong experience and perspective. Hepatobiliary Surg Nutr 2017;6:222-9. 